\title{
Simulation of LWIR TW ultrashort pulses over kilometer ranges in the atmosphere
}

P. Panagiotopoulos, P. Rosenow, K. Schuh, M. Kolesik, E. Wright, et al.

P. Panagiotopoulos, P. Rosenow, K. Schuh, M. Kolesik, E. M. Wright, S. W. Koch, J. V. Moloney, "Simulation of LWIR TW ultrashort pulses over kilometer ranges in the atmosphere," Proc. SPIE 10638, Ultrafast Bandgap Photonics III, 106381L (8 May 2018); doi: 10.1117/12.2306055

SPIE. Event: SPIE Defense + Security, 2018, Orlando, FL, United States 


\title{
Simulation of LWIR TW ultrashort pulses over kilometer ranges in the atmosphere
}

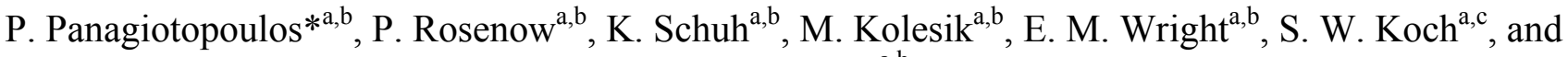 \\ J. V. Moloney ${ }^{\mathrm{a}, \mathrm{b}}$ \\ ${ }^{a}$ College of Optical Sciences, University of Arizona, Tucson, Arizona 85721, USA \\ ${ }^{\mathrm{b}}$ Arizona Center for Mathematical Sciences, University of Arizona, Tucson, Arizona 85721, USA \\ ${ }^{\mathrm{c}}$ Department of Physics and Materials Science Center, Philipps-University, 35032 Marburg, \\ Germany
}

\begin{abstract}
We have identified major paradigm shifts relative to near-IR filamentation when high power multiple terawatt laser pulses are propagated at mid-IR and long-IR wavelengths within key atmospheric transmission windows. Individual filaments at near-IR $(800 \mathrm{~nm})$ wavelengths typically persist only over tens of centimeters, despite the whole beam supporting them being sustained over about a Rayleigh range. In the important mid-IR atmospheric window (3.2 - 4 $\mu \mathrm{m}$ ) optical carrier wave self-steepening (carrier shocks) tend to dominate and modify the onset of long range filaments. These shocks generate bursts of higher harmonic dispersive waves that constrain the intensity growth of the filament to well below the traditional ionization limit, making long range low loss propagation possible. For long wavelength pulses in the 8-12 $\mu \mathrm{m}$ atmospheric transmission window, many-electron dephasing collisions from separate gas species act to dynamically suppress the traditional Kerr self-focusing lens and leads to a new type of whole beam self-trapping over multiple Rayleigh ranges. This prediction is key, since strong linear diffraction at these wavelengths are the major limitation and normally requires large launch beam apertures. We will present simulation results that predict multiple Rayleigh range propagation paths for whole beam self-trapping and will also discuss some recent efforts to extend the HITRAN linear atmospheric transmission/refractive index database to include nonlinear responses of important atmospheric molecular constituents.
\end{abstract}

Keywords: mid-IR, long-IR, filamentation, carrier, shock, atmosphere, many-body, HITRAN

\section{INTRODUCTION}

In recent year there have been concerted efforts worldwide to develop midwave (MWIR) and longwave (LWIR) high energy ultrashort laser pulses (USPs) targeting the important 3-5 $\mu \mathrm{m}$ and 8-12 $\mu \mathrm{m}$ atmospheric transmission windows. Linear LWIR sources in the 8-12 $\mu \mathrm{m}$ transmission window are known to show enhanced propagation through fog, atmospheric turbulence and cloud formations relative to mid-IR, visible and UV wavelengths. The potential impact of such sources for long range propagation applications is currently restricted by their short Rayleigh range and the consequent requirement for relatively large apertures at launch. By increasing the energy content of MWIR and LWIR ultrashort laser pulses, the hope is that the third order susceptibility of air can counteract the diffraction of the beam and create remote filaments sustained over extended paths. Currently available $3.9 \mu \mathrm{m}-80 \mathrm{fs}$ femtosecond sources with energies up to $25 \mathrm{~mJ}$ in energy ${ }^{1}$ which is around half the predicted required energy to sustain a high-power single filament over multiple tens of meters ${ }^{2}$. In stark contrast to the LWIR filamentation regime, at $800 \mathrm{~nm}$ individual filaments persist over a meter range. Current sources in the important 8-12 $\mu \mathrm{m}$ window with sufficient energy to potentially create a filamentation in air are found in the form of $\mathrm{CO}_{2}$ gas lasers with duration $\sim 3 \mathrm{ps}$ and with $\sim 3 \mathrm{~J}$ of energy have been reported ${ }^{3}$.

Recently, we reported that the fundamental physics of USP propagation is significantly changed at longer wavelengths $^{2,4,5}$. In particular, the well-known critical self-focusing (collapse singularity) which leads to extreme local

Ultrafast Bandgap Photonics III, edited by Michael K. Rafailov, Proc. of SPIE Vol. 10638,

$106381 \mathrm{~L} \cdot$ (c) 2018 SPIE · CCC code: 0277-786X/18/\$18 · doi: 10.1117/12.2306055 
intensities at $800 \mathrm{~nm}^{6,7}$ while still evident, is no longer regularized (arrested) through plasma defocusing. Instead, it is predicted that an optical carrier-wave shock develops early on, well before the collapse singularity is established and acts to limit the intensity growth by emitting recurrent bursts of dispersive waves that generate a broad featureless supercontinuum $^{2,8}$. Ionization through tunneling and multi-photon ionization now plays a relatively minor role and primarily acts to soften the extreme intensity spikes through additional plasma dispersion. Classical nonlinear envelope models, by default, fail to capture the physics in this regime. The appropriate mathematical canonical description can now be expressed in terms of a full electro-magnetic field resolved modified Kadomtsev-Petviashvili equation ${ }^{4}$. The latter explicitly exhibits two singularities (i) the well-known two dimensional critical collapse singularity, and (ii) an optical carrier-wave shock, where the shock velocity depends inversely on the local amplitude of the electric field, regularized by chromatic dispersion.

The challenge in creating long-lived filaments using MWIR or LWIR USPs lies in the growth of the critical power as $\lambda^{2}$. Consequently, the required power for self-focusing at $10 \mu \mathrm{m}$ for a given energy and pulse duration is a factor of $\sim 100$ larger than at $1 \mu \mathrm{m}$. Moreover, as we will show later, the highly detuned nature of the interaction at this long wavelength, brings the polarizability of the very weakly ionized, weakly interacting electrons associated with different ionized species in the gas into play. The latter will result in an ultrafast dynamic negative lensing effect which acts nonuniformly in the temporal domain and suppresses the optical Kerr self-focusing. This will produce a filament-like propagating wavepacket that can sustain a wide centimeter scale self-trapped beam waist over multiple Rayleigh ranges. Finally, parallel recent advances in first principles quantum calculations of the optical nonlinear response of various inert gases now allows us minimize ad hoc phenomenological modeling of light-matter coupling and capture self-focusing, multiphoton or tunneling ionization within a single self-consist theory.

For the strongly off-resonant MWIR and LWIR excitation conditions, the light-matter coupling is in the regime of ultrafast adiabatic following where the optically induced polarization is slaved by the instantaneous values of the exciting light field. In the absence of dephasing effects, the occupation of the excited state follows adiabatically the

exciting field, i.e. $f_{k}=E^{2}$. Thus, the system returns at the end of the excitation pulse into the ground state without any ionization. Clearly, strong-field ionization based on the interaction of single particles with the exciting field (multiphoton ionization, above threshold ionization or tunnel ionization) can break this adiabatic following. However, these effects require very high intensities in order to be efficient. An alternative way for the escape from adiabatic following is provided by many-body Coulomb interactions. In the field of optically excited semiconductors, it has been well established that the Coulombic scatterings of the optically induced microscopic polarizations can lead to dephasing. Since these processes depend on the degree of excitation in the system, these effects have been labeled as excitation induced dephasing (EID). Detailed experiment-theory comparisons have shown that these effects to dominate lightmatter coupling under suitable conditions. Even though the effects of EID are well-known in semiconductors, they are generally considered to be of lesser importance in atomic or molecular gases because these systems are dilute in comparison to solid-state densities. However, one should keep in mind that the decisive matrix element governing the strength of many-body scattering effects is given by the square of the active Coulomb potential. We predict that these many-body Coulomb weakly correlated quasi-free electrons while evident at MWIR wavelengths, dominate the nonlinear response at LWIR wavelengths (10 $\mu \mathrm{m}$ and longer).

\section{WAVEPACKET PROPAGATION MODELS}

\subsection{Nonlinear Schrödinger Equation model}

The nonlinear Schrödinger equation is widely recognized as the workhorse model for numerical pulse propagation in transparent media, especially in the extensively used spectral region spanning from UV to near-IR wavelengths ${ }^{7}$.

The model equation which describes the evolution of the slowly varying electric field envelope $\mathrm{E}_{\text {Env }}$ along $\mathrm{z}$ follows:

$$
K \frac{\partial E_{E n v}}{\partial z}=\frac{i}{2}\left[\nabla_{\perp}^{2} E_{E n v}+D E_{E n v}\right]+k_{0}\left[T^{2} N_{K e r r}\left(E_{E n v}\right)+T N_{N L L}\left(E_{E n v}\right)+N_{\text {Plasma }}\left(E_{E n v}, \rho\right)\right]
$$


Eq.(1) takes into account both linear (first term on the RHS) and nonlinear effects (second term on the RHS) that are acting on the electric field envelope $\mathrm{E}_{\mathrm{Env}} \cdot \nabla_{\perp}^{2}=\Delta_{\perp}=\frac{\partial^{2}}{\partial x^{2}}+\frac{\partial^{2}}{\partial y^{2}}$ is the transverse Laplacian operator which is used to model diffraction, while $\mathrm{D}$ is the dispersive operator used to model the material chromatic dispersion. The operators $K=k_{0}+i k_{0}^{\prime} \frac{\partial}{\partial t}$, and $T=1+i \omega_{0}^{-1} \frac{\partial}{\partial t}$ account for space-time focusing and self-steepening of the laser pulse.

The nonlinear source terms $\mathrm{N}_{\text {Kerr }}, \mathrm{N}_{\mathrm{NLL}}, \mathrm{N}_{\text {Plasma }}$ on the RHS of Eq.(1) are associated with the optical Kerr effect, nonlinear losses from MPA and plasma related effects like plasma defocusing and absorption.

While the nonlinear Schrödinger equation is more than adequate for modeling the propagation of pulses of wavelengths spanning from the UV to the near-IR, we will show that it unable to properly describe the propagation of intense pulses in the mid-IR and long-IR regime where carrier-wave related effects become important.

\subsection{Unidirectional Pulse Propagation Equation}

The unidirectional pulse propagation equation (UPPE) model $^{9,}{ }^{10}$, is essentially a unidirectional Maxwell propagator that fully resolves the underlying optical carrier-wave in the forward direction. Despite the higher computational requirements of such an approach, the resulting spectral solver shows close ideal parallel scaling on our in-house SGI UV2000 machine enabling propagation of radial symmetric simulations over multiple kilometers in a matter of a few hours. Results presented throughout this work are generated using the unidirectional pulse propagation equation (UPPE) model in both radial symmetry and $x y z t$ resolved numerical grids.

Since UPPE is a fully spectral electric field resolving propagator, the native representation of the laser field is expressed in arrays representing spectral amplitudes which are functions of angular frequency $\omega$, and transverse wavenumbers $\mathrm{k}_{\mathrm{x}}, \mathrm{k}_{\mathrm{y}}$. The spectral nature of the solver makes it possible to solve the linear propagation problem exactly. For a given medium permittivity $\varepsilon(\omega)$, the linear propagation properties are encoded in the propagation constants of the modal fields. In the case of a homogeneous medium these are plane waves, and the propagation constant depends on the angular frequency and the transverse wavenumbers:

$$
k_{z}\left(\omega, k_{x}, k_{y}\right)=\sqrt{\frac{\omega^{2} \varepsilon(\omega)}{c^{2}}-k_{x}^{2}-k_{y}^{2}}
$$

The permittivity is complex, consequently both chromatic dispersion and absorption are accounted both for exactly. This is major advantage of the UPPE model since in our case studies the spectral windows are enormous and need to be accurately resolved.

The polarization $\vec{P}(x, y, z, t)$ and current density $\vec{J}(x, y, z, t)$ are given as functions of the electric field $\vec{E}(x, y, z, t)$, thus the medium response is calculated from the history of the electric field independently at each spatial point of the numerical grid.

The exact system of equations describing the evolution of modal amplitudes along the z-axis for the forward and backward propagating field is given below. Eq.(3) describes the forward propagating wave

$$
\partial \vec{E}_{k_{x}, k_{y},+}^{\perp}(\omega, z)=+i k_{z} \vec{E}_{k_{x}, k_{y},+}^{\perp}(\omega, z)+\sum_{s=1,2} \vec{e}_{s}^{\perp} \vec{e}_{s}\left(\frac{i \omega^{2}}{2 \varepsilon_{0} c^{2} k_{z}} \vec{P}_{k_{x}, k_{y}}(\omega, z)-\frac{\omega}{2 \varepsilon_{0} c^{2} k_{z}} \vec{J}_{k_{x}, k_{y}}(\omega, z)\right)
$$

And Eqn.(4) describes the backward propagating wave 


$$
\partial \vec{E}_{k_{x}, k_{y},-}^{\perp}(\omega, z)=-i k_{z} \vec{E}_{k_{x}, k_{y},-}^{\perp}(\omega, z)-\sum_{s=1,2} \vec{e}_{s}^{\perp} \vec{e}_{s}\left(\frac{i \omega^{2}}{2 \varepsilon_{0} c^{2} k_{z}} \vec{P}_{k_{x}, k_{y}}(\omega, z)-\frac{\omega}{2 \varepsilon_{0} c^{2} k_{z}} \vec{J}_{k_{x}, k_{y}}(\omega, z)\right)
$$

Equations (3) and (4) are the coupled unidirectional pulse propagation equations (UPPE) ${ }^{10}$, while $\vec{e}_{1}$ and $\vec{e}_{2}$ are the unit vectors that are transverse to the propagation direction.

Equations (3) and (4) are mutually coupled through the polarization and current density terms. In order to solve the system, both the forward and backward propagating electric field are required. At this point we basic approximation of the UPPE model is made, i.e., that an accurately enough medium response can be calculated by the forward portion only:

$$
\vec{P}\left(\vec{E}_{-}+\vec{E}_{+}\right), \vec{J}\left(\vec{J}_{-}+\vec{E}_{+}\right) \stackrel{\text { UPPE approx. }}{\longrightarrow} \vec{P}\left(\vec{E}_{+}\right), \vec{J}\left(\vec{E}_{+}\right)
$$

Hence the canonical form of the scalar now UPPE can be written as follows:

$$
\partial_{z} E_{k_{x}, k_{y}}(\omega, z)=i K\left(k_{x}, k_{y}, \omega\right) E_{k_{x}, k_{y}}(\omega, z)+i Q\left(k_{x}, k_{y}, \omega\right) P_{k_{x}, k_{y}}(\omega, z)
$$

Where the exact linear propagator is

$$
K\left(k_{x}, k_{y}, \omega\right)=\sqrt{\frac{\omega^{2} \varepsilon(\omega)}{c^{2}}-k_{x}^{2}-k_{y}^{2}}
$$

And the nonlinear response term is given by

$$
Q\left(k_{x}, k_{y}, \omega\right)=\frac{\omega^{2}}{2 \varepsilon_{0} c^{2} \sqrt{\frac{\omega^{2} \varepsilon(\omega)}{c^{2}}-k_{x}^{2}-k_{y}^{2}}}
$$

This scalar UPPE model has been shown to be remarkably robust ${ }^{11}$ against spontaneously generated backward generated waves. Additionally the UPPE model is ideal in describing the MWIR and LWIR USP pulse propagation regime in various gaseous media. This is best reflected by comparing the UPPE model to the Nonlinear Envelope equation (NEE) model described in the previous section. The failure of NEE is illustrated in Figure 1 where a dramatic departure between UPPE and NEE becomes evident after initial self-focusing has set in. The simulation is for a $100 \mathrm{fs}$ duration $3.9 \mu \mathrm{m}$ pulse in dry air. The combined action of the optical carrier shock and critical self-focusing act in tandem to limit the intensity growth in the UPPE case. On the other hand, the envelope model is, by default, unable to capture the basic physics driving the propagation in this regime, and will start to blow-up. There simply is no physics based justification to assume that NEE or any nonlinear envelope variant of NEE can properly capture the physics at these longer wavelengths where carrier-wave driven effects are .

Figure 2 shows a "phase diagram" reflecting the operative physics when the wavelength increases from $800 \mathrm{~nm}$ to 8 $\mu \mathrm{m}$. The vertical intensity axis indicates achievable peak intensities in typical filaments. There are two regions that exhibit fundamentally different physical behavior and consequently greatly impact the propagation characteristics of 
USPs. For combinations of intensity-wavelengths that are in the black shaded region we predict the classical critical selffocusing (critical collapse) behavior characteristic of $800 \mathrm{~nm}$ filaments. In this region, linear dispersion of the relevant

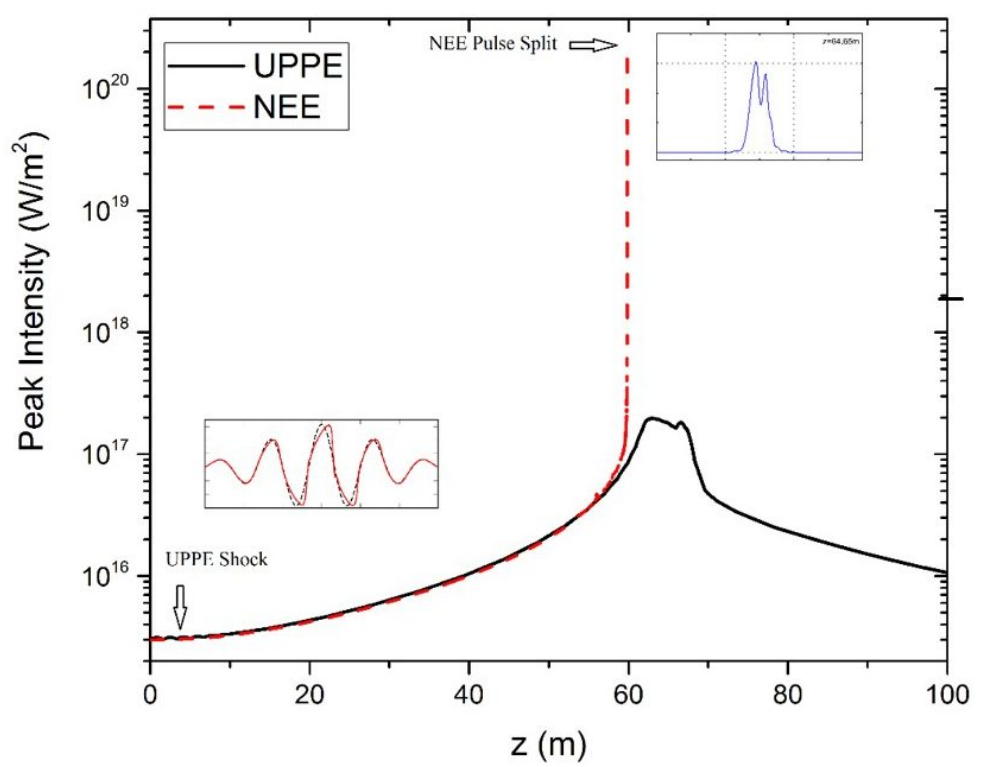

Figure 1. Comparison of the evolution of an identical initial $4 \mu \mathrm{m}$ pulses via the UPPE and the NEE. Lower inset on the left contrasts the strong optical carrier shock with a sinusoidal waveform after $\sim 5 \mathrm{~m}$. Upper inset on the top right shows the eventual splitting of the NEE envelope due to weak group velocity dispersion with the pulse dispersing subsequently.

species dominates over Kerr nonlinearity - In other words, the dispersion length is shorter than the characteristic nonlinear length scale which depends on the strength of the Kerr coefficient. Here physics of USP propagation is effectively captured by the classical Nonlinear Schrödinger equation (NLSE) and its many nonlinear envelope variants.

On the other hand, in the blue shaded region to the right which spans all wavelengths longer than about $3.5 \mu \mathrm{m}$ for intensities typically seen in filaments, the physics changes fundamentally. Here, the characteristic nonlinear length scale is shorter than the dispersion length scale. Now a new singularity emerges in addition to the critical self-focusing collapse. The underlying optical carrier-wave no longer remains sinusoidal and starts to self-steepen generating an electromagnetic shock wave.

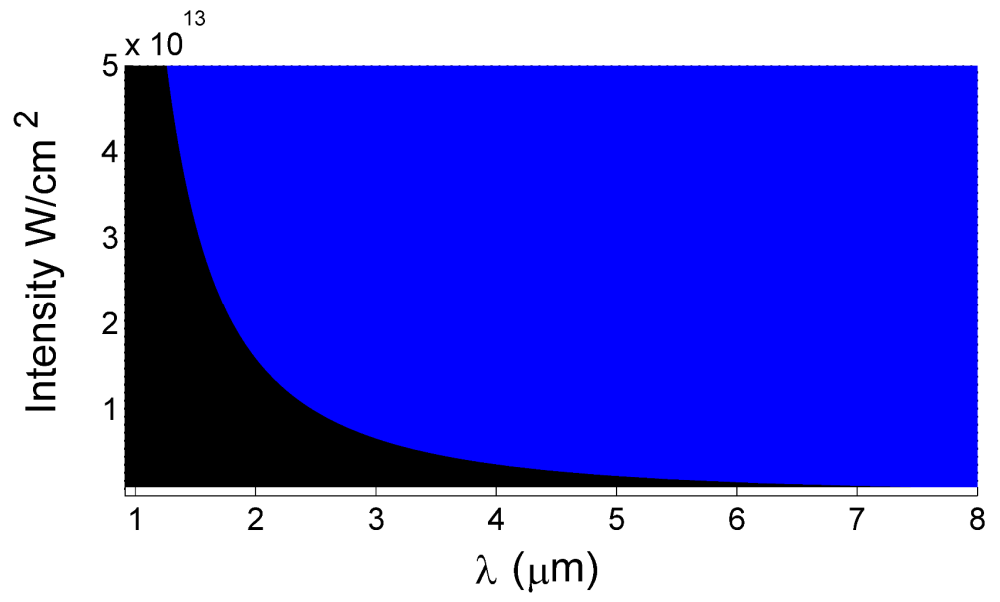

Figure 2. Phase diagram showcasing the separation between nonlinear envelope (black region) and UPPE (blue region) behavior. 


\section{ATMOSPERIC PROPAGATION OF 4 MICRON PULSES}

\subsection{Simple model for atmospheric optical properties}

In this work we employ the scalar UPPE for all simulations of USPs in the MWIR and LWIR. For this first approach we use a simple model for air in an attempt to showcase the basic physical mechanism driving USP in the midIR optical regime.

We model dry air taking into account chromatic dispersion based on the Sellmeier formula as parameterized by Peck and Reeder ${ }^{12,13}$. The real part of the linear susceptibility is depicted in Figure 3. The nonlinear refractive index is taking into account the instantaneous electronic response of $\mathrm{n}_{2}=9 \times 10^{-24} \mathrm{~m}^{2} / \mathrm{W}$ which was extrapolated by measurements ${ }^{14}$ from Milchberg's group at $800 \mathrm{~nm} \cdot \chi^{5}$ and other higher order nonlinearities are not taken into account. Ionization was modeled using the Keldysh formulation with an effective power law of $6.7 \times 10^{-138} \times \mathrm{I}^{8.5}$. We assume 2.5 x $10^{25}$ particles per cubic meter, only accounting for single electron ionization of Oxygen with a gap of $12.03 \mathrm{eV}$.

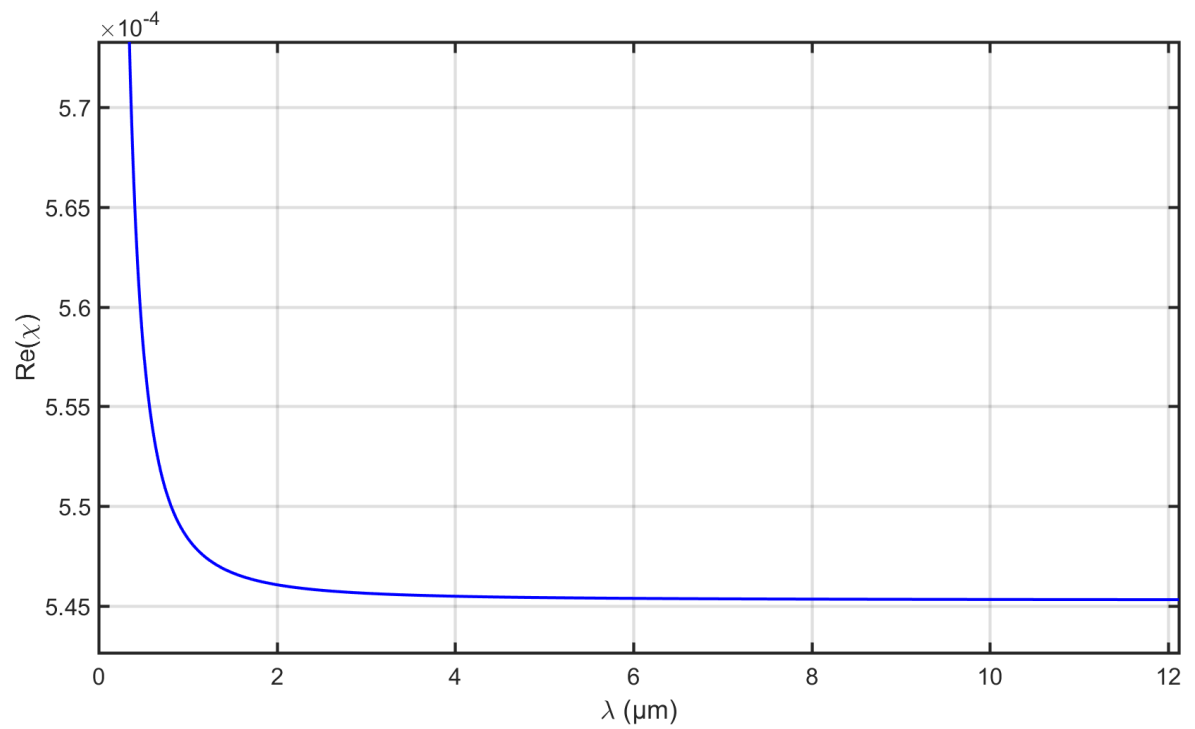

Figure 3. Real part of the linear susceptibility of air based on the classical Sellmeier equation ${ }^{12,13}$.

The initial wavepacket used in the simulation has a duration of $24 \mathrm{fs}$ at FWHM, carrying $177 \mathrm{~mJ}$ of energy at a central wavelength of $4 \mu \mathrm{m}$. The initial beam has a waist of $1.5 \mathrm{~cm}$ and is collimated. Our choice of wavelength is motivated by the flatness of the dispersive curve and the relative high transparency of the atmosphere in this specific spectral region. In addition to that, the ionization losses are expected to be much lower than at shorter wavelengths, making this wavelength ideal to explore long distance mid-IR filamentation.

Our simulations predict a new filamentation paradigm that is in stark contrast to classical $800 \mathrm{~nm}$ filaments. Our results for our $4 \mu \mathrm{m}-24 \mathrm{fs}-1.5 \mathrm{~cm}$ pulse are depicted in detail in Figure 4. In the classical picture of $800 \mathrm{~nm}$ filamentation, the non-saturated nonlinearity of the optical Kerr effect leading to a spatio-temporal collapse is arrested by plasma absorption and defocusing. However in the mid-IR regime studied here, a carrier shock is formed that leads to collapse-accelerated self-steepening of the optical carrier-wave, well before ionization becomes relevant. The steep shock front generates an enormous supercontinuum that encompasses all even and odd harmonics. These being at shorter wavelengths, propagate as wavepackets away from the fundamental at a different group velocity. Group velocity dispersion, although weak, is critically important in regularizing the collapse in his way. The result is an intense light bullet that is sustained for extended propagation distances driven by multiple recurrent carrier wave shock steepening 

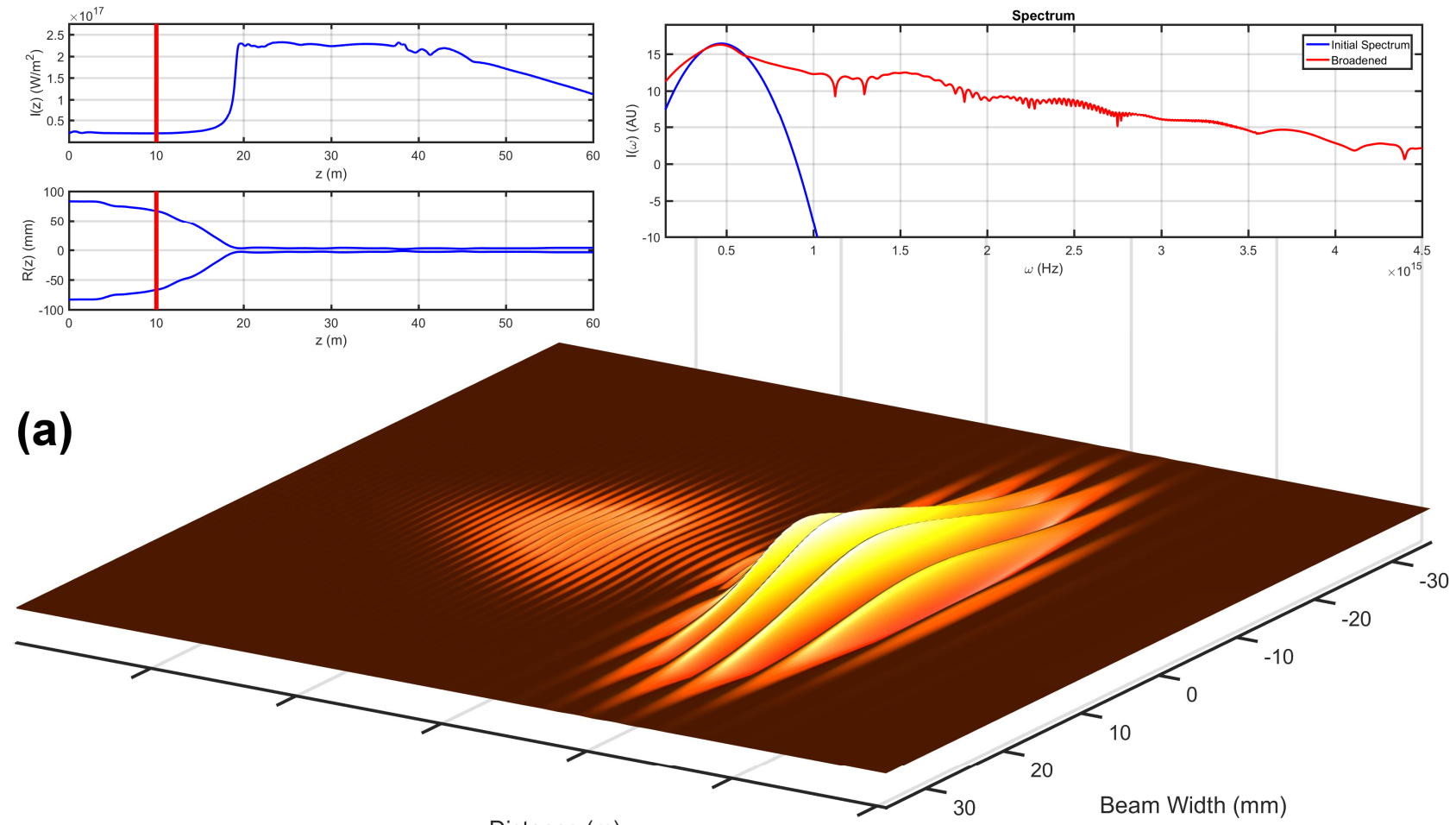

Distance $(m)$
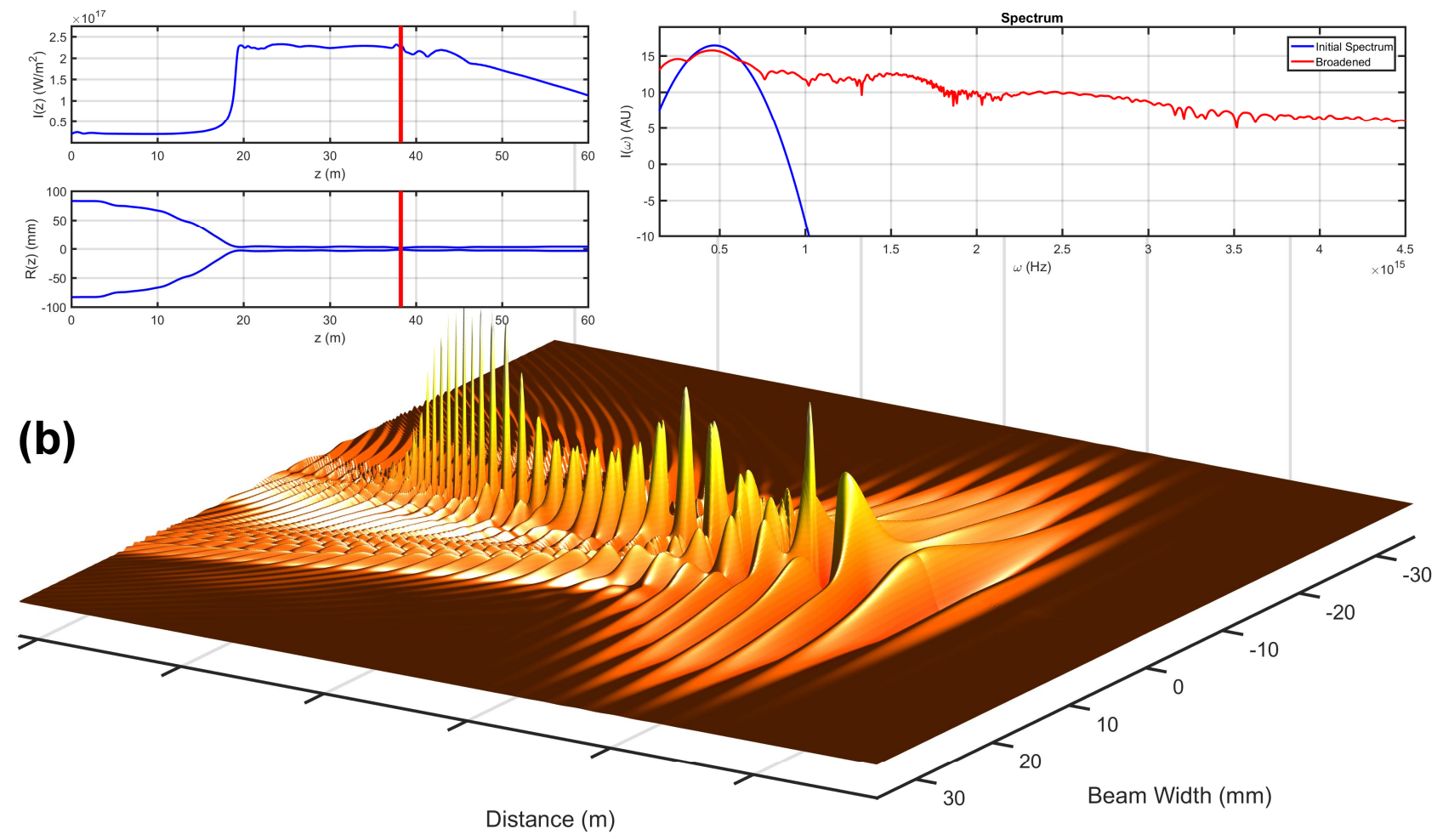

Figure 4. Showcase of the new filamentation paradigm found in the MWIR and LWIR regime in the atmosphere. Spatiotemporal representation of the electric field of the $4 \mu \mathrm{m}$ pulse at (a) $\mathrm{z}=10 \mathrm{~m}$ and (b) $\mathrm{z}=38 \mathrm{~m}$. Top insets show the peak intensity and beam waist vs propagation distance $\mathrm{z}$, and the corresponding spectrum at each distance. The red vertical lines indicate the position in $\mathrm{z}$ that is depicted in the surface on the bottom. 
events, which in turn initiate bursts of spectrally broadened emissions at the back of the main pulse that act to temporally soften the shock front and arrest the collapse. Notably, this collapse arresting mechanism is spectral/temporal in contrast to the classical Kerr vs plasma filamentation paradigm that is mainly acting in the spatial domain.

The resulting mid-IR filament is much thicker $(\sim 1 \mathrm{~mm})$ than its $800 \mathrm{~nm}$ counterparts $(\sim 100 \mu \mathrm{m})$, and is able to extend over tens of meters in the atmosphere in a nearly lossless fashion $(\sim 15 \%$ losses of total energy over $100 \mathrm{~m})$. As can be seen in Figure 4., the leading edge of the electric field is remaining almost unchanged over $30 \mathrm{~m}$ of propagation, while higher harmonic burst are constantly emitted from the tail of the wavepacket.

\section{ATMOSPERIC PROPAGATION OF 10 MICRON PULSES}

\subsection{Extended model for atmospheric optical properties: HITRAN dispersion and absorption}

Moving from $4 \mu \mathrm{m}$ pulse to $10 \mu \mathrm{m}$ pulses, we need to extend and refine our material model for air, since dispersion and absorption lines are now close to the $3^{\text {rd }}$ and higher harmonics of $10 \mu \mathrm{m}$. In our effort to refine our model we now include many quantities routinely ignored in theoretical filamentation studies, by utilizing the extensive HITRAN ${ }^{15}$ database. We assume the composition of air as $78.1 \% \mathrm{~N}_{2}, 20.9 \% \mathrm{O}_{2}, 0.9 \%$ Argon, $0.037 \% \mathrm{CO}_{2}, 1.8 \times 10^{-4} \% \mathrm{CH}_{4}$ and $10^{-5} \% \mathrm{CO}$. Only the most common isotope for each species is accounted for. In addition, water vapor is also included in our model for a modest $10 \%$ relative humidity (good optical atmospheric conditions). Temperature is $25 \mathrm{C}$. The linear optical properties of this system are calculated based on the formalism detailed in previous works ${ }^{16}$. The resulting real and imaginary parts of the complex linear susceptibility are depicted in Figure 5. (a) and (b) respectively for a wide spectral range. Notably, the black vs the other two lines only differ in the amount of water vapor concentration in the atmosphere which showcases the importance and impact even 10\% of water vapor has on the linear optical properties of air.
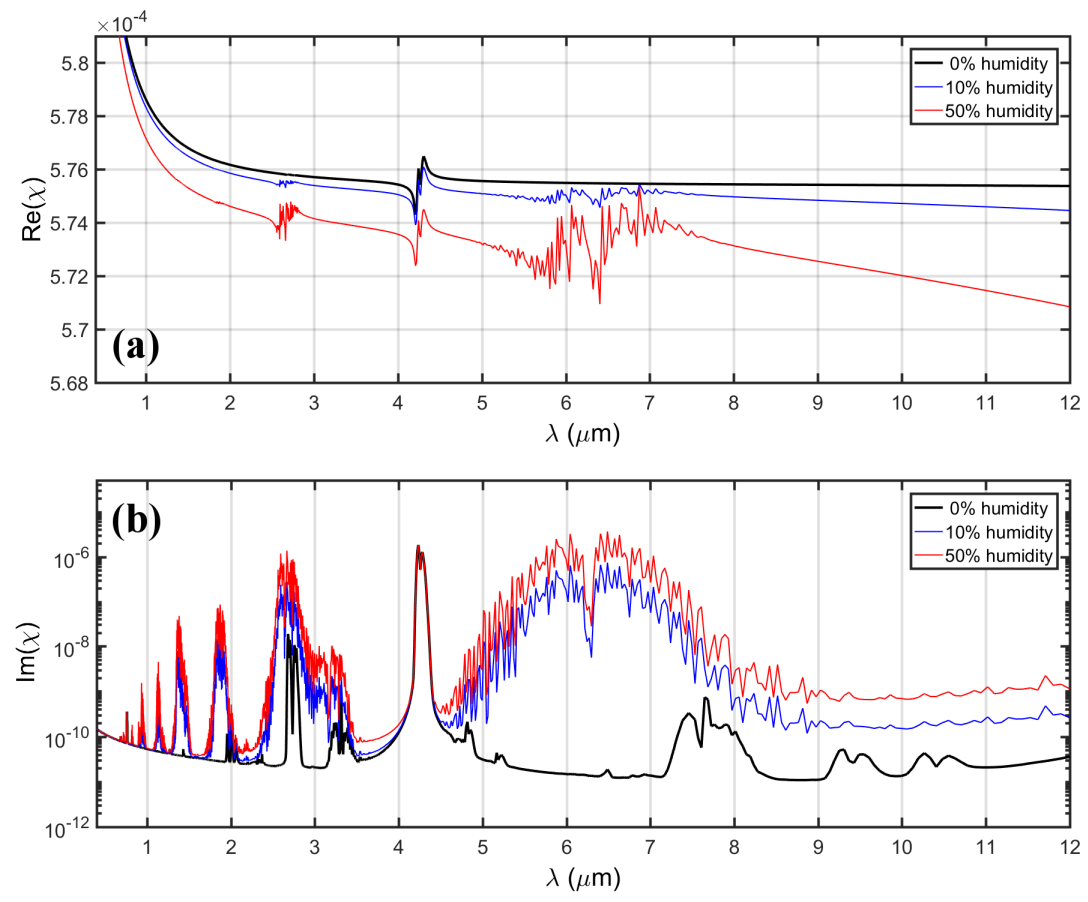

Figure 5. (a) Real and (b) imaginary parts of $\chi^{1}$ of the atmosphere as modeled by the HITRAN database for various humidity values at a temperature of $25 \mathrm{C}$ and $1 \mathrm{~atm}$. The black, blue, and red curves correspond to $0 \%, 10 \%$, and $50 \%$ relative humidity. The case of $10 \%$ relative humidity represents a good compromise of ideal, but still realistic, atmospheric conditions for long range laser propagation. 


\subsection{Extended model for atmospheric optical properties: Many-body interactions and excitation induced dephasing}

In addition to refining the atmospheric model for linear dispersion and absorption in the previous section, we have to also take into account many-body effects and excitation induced dephasing in this wavelength regime. As can be seen in Figure 6. the impact of many-body excitation induced dephasing is much more pronounced at $10 \mu \mathrm{m}$ than at $4 \mu \mathrm{m}$. The overall impact on beam curvature (black curve) is greatly modified when compared to the ionization-free case (black dashed curve). At $10 \mu \mathrm{m}$ we predict a strong suppression of the optical Kerr effect self-focusing lens due to manybody excitation induced dephasing, which should in principle smooth out the abrupt intensity spikes commonly observed in optical filaments.
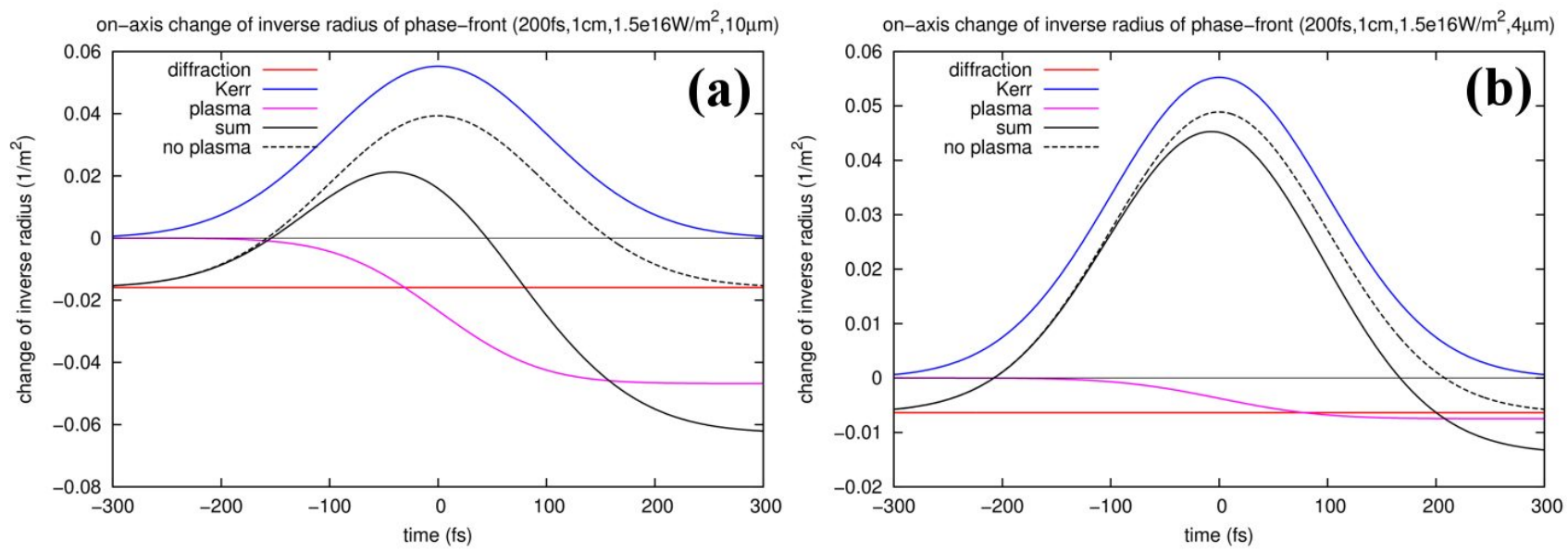

Figure 6. Impact of various physical effects on beam focusing for (a) $10 \mu \mathrm{m}$, (b) $4 \mu \mathrm{m}$ pulses vs time. Red curve: diffraction. Blue curve: optical Kerr effect. Magenta curve: ionization due to many body excitation induced dephasing. Black curve: sum of the above effects. Black dashed curve: sum of the above without EID. Calculation is based on Argon gas.

While the full study of the many-body excitation induced dephasing for the all species found in the atmosphere is still an ongoing work, we estimate that that Argon is a close qualitative approximation of air in this regard ${ }^{17}{ }^{18}$. The simplified many-body effects model can be described by the following equation

$$
\frac{d f}{d t}=c E^{4} \sqrt{\frac{E^{2}+s}{E^{2}}}
$$

Where $f$ is the spatio-temporal plasma density due to many-body interactions, and $\mathrm{E}$ the electric field of the laser pulse. The parameters $\mathrm{c}=2.84 \times 10^{-7}\left(\mathrm{mV}^{-4} \mathrm{~s}^{-1}\right)$ and $\mathrm{s}=4.6 \times 10^{18}\left(\mathrm{~V}^{2} \mathrm{~m}^{-2}\right)$ are fitted to the full microscopic results for Argon gas.

\subsection{Simulation result of $10 \mu \mathrm{m}$ pulses in realistically modeled atmosphere}

For our study of $10 \mu \mathrm{m}$ pulses we also included the delay rotational Raman response of Nitrogen and Oxygen as modeled by Palastro et. al. ${ }^{19}$. We split the optical Kerr effect into an instantaneous electronic response with nonlinear 
indices of $\mathrm{n}_{2, \text { Oxygen }}=8.3 \times 10^{-24} \mathrm{~m}^{2} / \mathrm{W}$ and $\mathrm{n}_{2 \text {,Nitrogen }}=1.67 \times 10^{-23} \mathrm{~m}^{2} / \mathrm{W}$, while the rotational response is modeled by using a damped harmonic oscillator with the natural frequency damping and steady state index chosen to fit the quantum mechanical density-matrix calculation ${ }^{19}$. Ionization is modeled using many-body effects as described in the previous section, while multi-photon ionization is considered negligible for our working intensities. Linear dispersion and absorption is modeled by using the full HITRAN dataset as described previously.

We use a $10 \mu \mathrm{m}$ Gaussian spatio-temporal wavepacket with beam waist of $3 \mathrm{~cm}$ at $1 / \mathrm{e}^{2}$ radius and a varying pulse duration from 2.4 ps to 3 ps. The wavepacket starts out collimated and without any input chirp, having a diffraction length of $283 \mathrm{~m}$ and peak intensity of $8.2 \times 10^{14} \mathrm{~W} / \mathrm{m}^{2}$, which corresponds to a peak power of $1.16 \mathrm{TW}$ (2.3 critical powers).
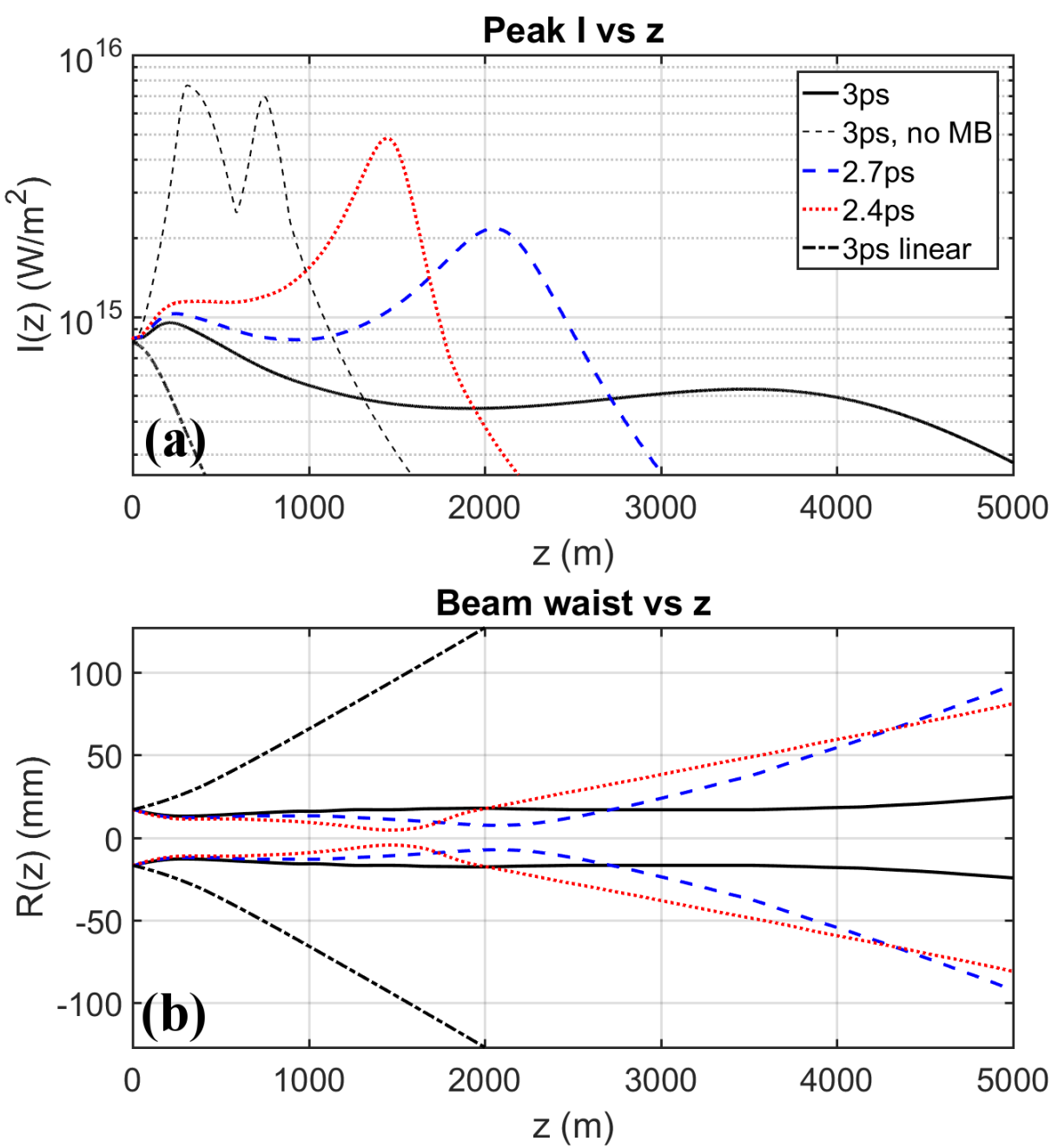

Figure 7. (a) Peak intensity and (b) beam waist, versus propagation distance $\mathrm{z}$ for $10 \mu \mathrm{m}$ pulses with initial intensities of 8.2 $\mathrm{x} 10^{14} \mathrm{~W} / \mathrm{m}^{2}, 3 \mathrm{~cm}$ beam waist and $3 \mathrm{ps}$ (black continuous curve), $2.7 \mathrm{ps}$ (blue dashed curve), and $2.4 \mathrm{ps}$ (red dotted curve) durations. The thin black dashed curve corresponds to the 3 ps pulse where many-body effects have not been taken into account. The black-dotted curve corresponds to the linear propagation of the 3 ps pulse. Figure taken from our recent published work ${ }^{20}$.

Figure 7. shows the result of our simulations over a $5 \mathrm{~km}$ propagation distance. We can see that for the optimal pulse duration of 3 ps, depicted as the black curves in Figure (a) and (b), an extremely long and uniform filament is created. This soliton-like optical structure is persisting over multiple Rayleigh ranges with an almost invariant beam 
waist and peak intensity. For shorter pulse durations, the filament becomes more intense and consequently less stable and overall shorter. This behavior is in line with the cumulative nature of the many-body ionization and excitation induced dephasing which is a cumulative effect in time. For shorter durations, the cumulative effect is not enough to suppress the Kerr lens and intensity rises to higher values, effective cutting the overall propagation distance short.

\subsection{Nonlinear HITRAN}

Beyond the linear contribution from rovibrational response, nonlinear contributions are to be expected for high enough intensities. In order to include these into simulations, we applied the optical Bloch equations to transition data from the HITRAN database ${ }^{21}$. Since the amount of data in HITRAN is rather prohibitive, a selection procedure based on transition strength and detuning from the carrier frequency was applied, reducing the number of transitions from more than 140,000 to 12,709 . The linear rovibrational response is dominated by rotational transitions below the carrier frequency, leading to a negative response overall. This is enforced by the nonlinear response to short pulses at increased intensities, with a reversal at very high intensities. The negative contribution to the polarization response is weaker for longer pulses. The overall properties of the polarization response indicate a weakened focusing due to rovibrational response.

For the purpose of propagation simulations, a simplified approached is applied within the UPPE framework. Here, correlations between transitions are neglected in order to speed up the response calculation. This approximation cannot describe coherent excitations that become relevant at high intensities and limits the applicability of this approach to intensities below $4 \times 10^{16} \mathrm{Wm}^{-2}$. The propagation results for pulses with a power of $1.6 \mathrm{TW}$ (corresponding to $1 \times 10^{16}$ $\mathrm{Wm}^{-2}$ initial peak intensity and slightly more than one critical power) and $3.2 \mathrm{TW}\left(2 \times 10^{16} \mathrm{Wm}^{-2}\right)$ are shown in Fig. $\mathrm{N}$ for a model atmosphere of argon with $1 \%$ water vapor. A comparison to a pure Argon atmosphere is given as well. It can be seen, that the presence of water and the resulting nonlinear rovibrational response lowers the focusing of the pulse compared to the water free case.

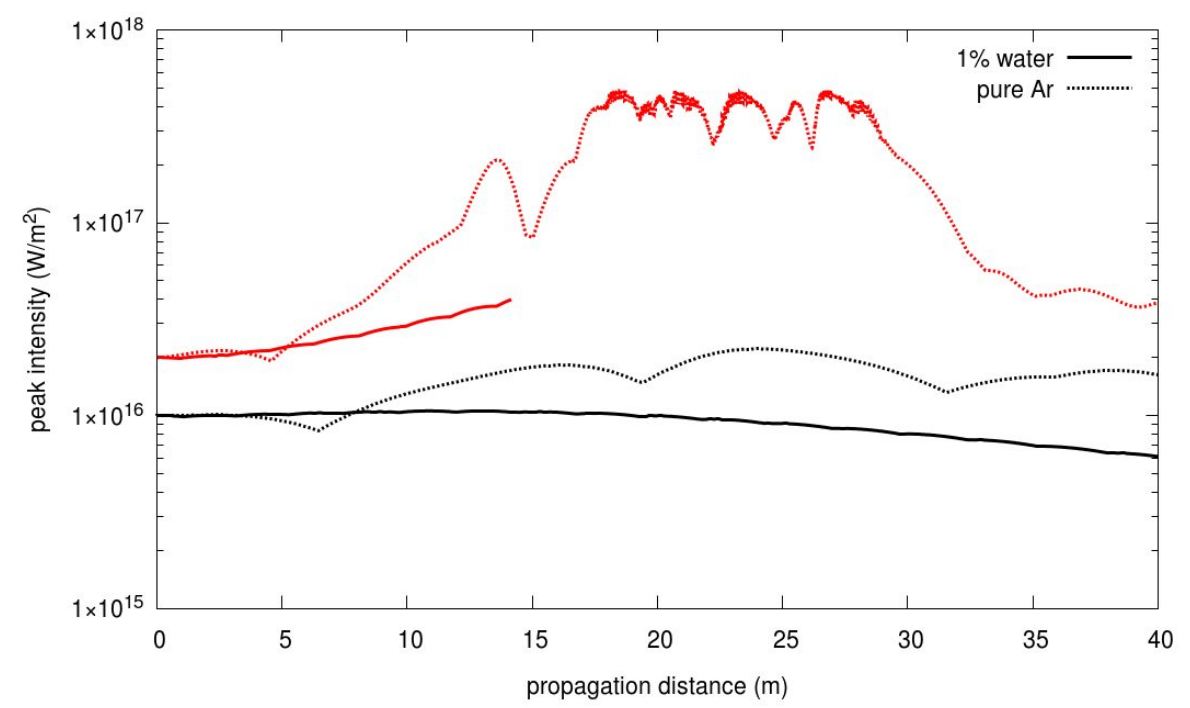

Figure 8. Peak intensity for a pulse with a power of 1.6 TW (black) and 3.2 TW (red) in an Argon-water model atmosphere and pure Argon for comparison. The $3.2 \mathrm{TW}$ pulse is cut off when the peak intensity goes above the range of applicability for the simplified model. 


\section{SUMMARY}

In conclusion, we have presented a new filamentation paradigm found at mid-IR and long-IR wavelengths capable of extremely long range propagation distance in the multi-TW, multi-Joule regime in the atmosphere. In stark contrast to the classical near-IR $(800 \mathrm{~nm})$ to UV regime, we find that MWIR USPs can still undergo filamentation but with filament waists on the order of a few millimeters. This allows one to pack more power and energy into a single filament and deliver it to more distant targets. For LWIR pulses in the important 8-12 $\mu \mathrm{m}$ atmospheric window, we predict that essentially infinite range, many-body Coulomb correlations between distant atoms/molecules in the gas can suppress a otherwise dominating self-focusing Kerr effect, resulting into wavepacket that can propagate, nearly lossless, over multiple kilometers in the atmosphere.

\section{ACKNOWLEDGMENTS}

Our work has been supported by an Air Force Office of Scientific Research Multidisciplinary University Research Initiative (MURI). Grant no: FA9550-16-1-0088. We also acknowledge the partial support through an ONR MURI grant N00014-17-1-2705.

\section{REFERENCES}

[1] A. V. Mitrofanov, A. A. Voronin, D. A. Sidorov-Biryukov et al., "Mid-infrared laser filaments in the atmosphere," Scientific Reports, 5, 8368 (2015).

[2] P. Panagiotopoulos, P. Whalen, M. Kolesik et al., "Super high power mid-infrared femtosecond light bullet," Nat Photon, 9(8), 543-548 (2015).

[3] D. Haberberger, S. Tochitsky, and C. Joshi, "Fifteen terawatt picosecond CO2 laser system," Optics Express, 18(17), 17865-17875 (2010).

[4] P. Whalen, P. Panagiotopoulos, M. Kolesik et al., "Extreme carrier shocking of intense long-wavelength pulses," Physical Review A, 89(2), 023850 (2014).

[5] P. Panagiotopoulos, M. Kolesik, and J. V. Moloney, "Exploring the limits to energy scaling and distant-target delivery of high-intensity midinfrared pulses," Physical Review A, 94(3), 033852 (2016).

[6] M. Mlejnek, M. Kolesik, J. V. Moloney et al., "Optically turbulent femtosecond light guide in air," Physical Review Letters, 83(15), 2938-2941 (1999).

[7] A. Couairon, and A. Mysyrowicz, "Femtosecond filamentation in transparent media," Physics Reports 441(24), 47-189 (2007).

[8] P. Panagiotopoulos, P. Whalen, M. Kolesik et al., "Carrier field shock formation of long-wavelength femtosecond pulses in single-crystal diamond and air," Journal of the Optical Society of America B, 32(8), 1718-1730 (2015).

[9] M. Kolesik, J. V. Moloney, and M. Mlejnek, "Unidirectional optical pulse propagation equation," Physical Review Letters, 89(28), 283902 (2002).

[10] M. Kolesik, and J. V. Moloney, "Nonlinear optical pulse propagation simulation: From Maxwell's to unidirectional equations," Physical Review E, 70(3), 036604 (2004).

[11] M. Kolesik, P. Jakobsen, and J. V. Moloney, "Quantifying the limits of unidirectional ultrashort optical pulse propagation," Physical Review A, 86(3), 035801 (2012).

[12] W. Sellmeier, "Zur Erklärung der abnormen Farbenfolge im Spectrum einiger Substanzen,” Ann. Phys. Chem., 219, 272-282 (1871).

[13] E. R. Peck, and K. Reeder, "Dispersion of Air,” J. Opt. Soc. Am., 62(8), 958-962 (1972).

[14] J. Wahlstrand, Y. H. Cheng, and H. Milchberg, "Absolute measurement of the transient optical nonlinearity in N2, O2, N2O, and Ar,” Physical Review A, 85(4), 043820 (2012). 
[15] L. S. Rothman, I. E. Gordon, Y. Babikov et al., “The HITRAN2012 molecular spectroscopic database,” Journal of Quantitative Spectroscopy and Radiative Transfer, 130, 4-50 (2013).

[16] R. J. Mathar, "Calculated refractivity of water vapor and moist air in the atmospheric window at $10 \mu \mathrm{m}$," Applied Optics, 43(4), 928-932 (2004).

[17] K. Schuh, J. V. Moloney, and S. W. Koch, "Interaction-induced nonlinear refractive-index reduction of gases in the midinfrared regime," Physical Review E, 93(1), 013208 (2016).

[18] K. Schuh, M. Kolesik, E. M. Wright et al., "Self-Channeling of High-Power Long-Wave Infrared Pulses in Atomic Gases," Physical Review Letters, 118(6), 063901 (2017).

[19] J. P. Palastro, T. M. Antonsen, S. Varma et al., "Simulations of femtosecond atmospheric filaments enhanced by dual pulse molecular alignment," Physical Review A, 85(4), 043843 (2012).

[20] K. Schuh, P. Panagiotopoulos, M. Kolesik et al., "Multi-terawatt $10 \mu \mathrm{m}$ pulse atmospheric delivery over multiple Rayleigh ranges," Optics Letters, 42(19), 3722-3725 (2017).

[21] K. Schuh, P. Rosenow, M. Kolesik et al., "Nonlinear rovibrational polarization response of water vapor to ultrashort long-wave infrared pulses," Physical Review A, 96(4), 043818 (2017). 